\title{
Systemic therapy in addition to cytoreduction and hyperthermic intraperitoneal chemotherapy for colorectal peritoneal metastases: recent insights from clinical studies and translational research
}

\author{
Checca Bakkers ${ }^{1}$, Geert A. A. M. Simkens ${ }^{1}$, Ignace H. J. T. De Hingh ${ }^{1,2}$ \\ ${ }^{1}$ Department of Surgery, Catharina Cancer Institute, Eindhoven, The Netherlands; ${ }^{2}$ GROW - School for Oncology and Development Biology, \\ Maastricht University, Maastricht, The Netherlands \\ Contributions: (I) Conception and design: All authors; (II) Administrative support: All authors; (III) Provision of study materials or patients: None; \\ (IV) Collection and assembly of data: None; (V) Data analysis and interpretation: None; (VI) Manuscript writing: All authors; (VII) Final approval of \\ manuscript: All authors. \\ Correspondence to: Ignace H. J. T. De Hingh, MD. Department of Surgery, Catharina Cancer Institute, PO Box 1350, 5602 ZA Eindhoven, The \\ Netherlands. Email: ignace.d.hingh@catharinaziekenhuis.nl.
}

\begin{abstract}
There is a lack of randomized or high-quality intention-to-treat cohort studies addressing the role of systemic therapy in addition to cytoreductive surgery and hyperthermic intraperitoneal chemotherapy (CRS-HIPEC) as part of the treatment of colorectal peritoneal metastases (PM). Therefore, the choice whether or not to treat patients with systemic therapy is currently mainly based on expert opinion. As a result, treatment with neoadjuvant and/or adjuvant systemic therapy is implemented in various ways around the world. The aim of this review was to provide an overview of recent insights with regard to the systemic treatment of PM of colorectal origin obtained from clinical studies and translational research.
\end{abstract}

Keywords: Systemic therapy; cytoreductive surgery (CRS); hyperthermic intraperitoneal chemotherapy (HIPEC); peritoneal metastases (PM); colorectal cancer

Submitted Feb 29, 2020. Accepted for publication Jun 04, 2020.

doi: 10.21037/jgo-20-133

View this article at: http://dx.doi.org/10.21037/jgo-20-133

\section{Introduction}

The peritoneum is the second most prevalent metastatic site of colorectal cancer (CRC). As a result of intraperitoneal seeding of cancer cells, peritoneal metastases (PM) can develop throughout the abdominal cavity. In about $5 \%$ of CRC patients, PM are diagnosed at time of diagnosis of the primary tumor (synchronous metastases) $(1,2)$. In another $\sim 5 \%$ of CRC patients, PM will develop after curative resection of the primary tumor (metachronous metastases) (3-5). These numbers are probably an underestimation of the real incidence, as PM are difficult to be diagnosed with imaging studies and indeed, autopsy studies report much higher incidences (6).

Nowadays, cytoreductive surgery with hyperthermic intraperitoneal chemotherapy (CRS-HIPEC) is considered standard treatment in patients with limited intraperitoneal disease in most countries $(7,8)$. Unfortunately, locoregional and/or systemic recurrence occurs in most patients, even after aggressive and complete cytoreduction and HIPEC. In $35-41 \%$ of all patients, this even occurs within 1 year after treatment $(9,10)$. In an effort to prevent or delay recurrence and thus prolong overall survival (OS), various experts have advocated systemic treatment in these patients either in a neoadjuvant setting, adjuvant setting or a combination of both. Others have questioned this strategy as compelling evidence to support systemic treatment in addition to CRSHIPEC is currently lacking and side-effects may be severe. In the absence of consensus amongst experts, there is a wide 
variation in systemic treatment protocols of these patients. The aim of the current review was to provide an overview of recent clinical and translational data with regard to systemic treatment of patients with PM of colorectal origin treated with CRS-HIPEC.

\section{The rationale of (neo)adjuvant systemic therapy}

There are various hypotheses that support the efficacy of systemic therapy in the context of CRS-HIPEC. Firstly, systemic therapy may eradicate micro metastases. With a high risk of systemic spread in advanced primary colorectal tumors, the addition of systemic therapy may lower the risk of distant metastases during and after treatment $(1,11,12)$. Secondly, neoadjuvant systemic therapy may lower the intraperitoneal tumor-load prior to surgery $(13,14)$. If the extent of disease decreases, the extent of surgery needed for complete resection is likely to decrease too. Besides a possible greater chance of complete resection, less extensive surgery may also lead to fewer post-operative complications $(15,16)$. Finally, administration of systemic chemotherapy prior to CRS-HIPEC may select patients that will respond more favorable to CRS-HIPEC (17-19). Response to neoadjuvant treatment provides insight in tumor behavior and might be helpful in surgeon's decision making on whether or not to continue with CRS-HIPEC (20-22).

Interestingly, similar arguments have been used in the past to treat patients undergoing surgery for colorectal liver metastases ( $\mathrm{LM}$ ) with perioperative systemic therapy. This strategy was investigated in the EPOC-trial and compared with surgical treatment alone $(23,24)$. Although this trial showed a significant improvement in progressionfree survival (PFS) in patients treated with perioperative systemic treatment as compared to patients who underwent surgery alone, there was no difference in OS between both groups.

\section{Potential risks of (neo)adjuvant systemic therapy}

Besides the listed possible benefits of adding systemic therapy to CRS-HIPEC, it may also involve risks. Most importantly and most concerning is the potential effect on patients' general condition, particularly in the neoadjuvant setting. Systemic treatment with chemotherapy $( \pm$ targeted therapy) and its consequent toxicity may significantly deteriorate patients' general condition (25). However, it is unknown to what extent this might lead to inoperability in patients with colorectal PM intended to undergo CRS-
HIPEC, as only one small study has investigated this in an intention-to-treat setting (26). Another risk is that selection of patients by response to neoadjuvant treatment may improve survival of those patients that will eventually undergo CRS-HIPEC, but patients that progress during systemic treatment may have been denied a potential beneficial treatment with CRS-HIPEC.

Furthermore, systemic treatment may lead to an increased risk of post-operative morbidity. The main concern in this is the addition of VEGF-inhibitors in the neoadjuvant setting, as one study demonstrated that the administration of neoadjuvant VEGF-inhibitors increased the risk of post-operative complications by its possible anti-angiogenetic effects on healing processes such as anastomotic healing and wound healing (27). Finally, the addition of (neo)adjuvant systemic therapy to the treatment of patients with colorectal PM prolongs and intensifies treatment. This may temporarily or permanently affect quality of life (QoL). Currently, the effect of the addition of systemic treatment to CRS-HIPEC on QoL and costs is unknown (28).

\section{Insights from clinical studies}

In 2017, two systematic reviews addressing the role of (neo) adjuvant systemic therapy in patients with colorectal PM undergoing CRS-HIPEC were published. Both Rovers et al. (20) and Waite et al. (29) concluded that available evidence on the value of (neo)adjuvant systemic therapy as part of the treatment of colorectal PM was limited. There were no randomized controlled trials (RCT's) available and heterogeneity within the listed prospective and retrospective observational cohort studies was evident. None of the cohort studies were performed according to an adequate intentionto-treat design. Rovers et al. concluded that, despite the absence of high-level evidence, neoadjuvant systemic therapy might result in improved survival in selected patients. The value of adjuvant systemic therapy was questioned. However, Waite et al. concluded that there is some low-level evidence suggesting that adjuvant chemotherapy prolongs OS. Both study groups concluded that high-quality data investigating this topic are urgently needed.

Since the publication of these two systematic reviews, only one prospective study on this subject has been published: the COMBATAC trial (26). In this multicenter, open-label, single-arm study, patients with synchronous and metachronous colorectal or appendiceal PM were included and were treated with neoadjuvant systemic 
therapy (doublet chemotherapy and cetuximab), followed by CRS-HIPEC. Unfortunately, the study was prematurely terminated because of insufficient accrual. However, results of this study are very interesting since this trial is the first to provide insight in the neoadjuvant treatment of patients with colorectal PM in an intention-to-treat setting. In this trial, a significant drop-out rate of patients during neoadjuvant systemic therapy was observed: of 25 patients undergoing neoadjuvant treatment, eventually only 14 patients underwent CRS-HIPEC. This is an interesting finding as most studies only report on patients that received systemic treatment and subsequently underwent CRSHIPEC. By doing so, a selection bias is introduced and the reported survival results usually do not reflect the true impact of systemic therapy and CRS-HIPEC, as the outcomes of patients that drop-out during neo-adjuvant therapy are not taken into account. Such selection bias is probably also partially underlying the remarkably long survival (about 42 months) in both arms of the recently presented PRODIGE-7 trial (30). In this trial, patients were only included if they underwent at least 6 cycles of systemic treatment and underwent CRS-HIPEC. This phenomenon questions the external validity of this trial as no data were presented on the intention-to-treat group, consisting of all patients that were diagnosed with PM before undergoing (any) treatment.

To investigate the effect of systemic treatment in an RCT, the Dutch CAIRO6 trial was initiated in 2017 and is currently recruiting patients in all HIPECcenters in the Netherlands. In this trial, patients with resectable synchronous or metachronous colorectal PM are randomized (1:1) for upfront CRS-HIPEC without (neo)adjuvant systemic therapy (group A) or CRSHIPEC, preceded by neoadjuvant systemic therapy and followed by adjuvant systemic therapy (group B). In group $\mathrm{B}$, the neoadjuvant systemic treatment comprises four cycles of capecitabine with oxaliplatin (CAPOX) or six cycles of 5 -fluorouracil/leucovorin with oxaliplatin (FOLFOX) or six cycles of 5 -fluorouracil/leucovorin with irinotecan (FOLFIRI), with bevacizumab. In the adjuvant setting, another 4-6 cycles of systemic therapy (without bevacizumab) are administered if patients are considered suitable (31). This intention-to-treat trial is subdivided in a phase II and a phase III study. The phase II study focuses on safety and feasibility of perioperative systemic treatment and was completed early 2019. The primary endpoint of the phase III trial, which is currently recruiting patients, is 3 -year OS, with the hypothesis of a $15 \%$ increase in OS in group $\mathrm{B}$, as compared to group $\mathrm{A}$.

\section{Insights from translational studies}

There is a growing understanding that both the indication for and the type of systemic treatment in CRC patients should no longer be based on traditional clinical parameters such as tumor stage and lymph node status alone. Other factors such as the mutational status of the tumor, microsatellite (in)stability, sidedness of the tumor (left versus right), age and gender of the patient should be considered as well. Recent studies have shown that tumor specific properties may be of importance in the treatment of $\mathrm{PM}$ as well.

\section{Consensus molecular subtypes in CRC}

In 2015, a collaboration of six study groups who had previously developed a methodology for CRC classification based on gene-expression was established (32). Combining the six subtyping algorithms resulted in the "Consensus Molecular Subtype classification". This classification comprises 4 subtypes in total (CMS1-4), each of which having specific biological behavior and subsequent implications for optimal treatment and prognosis. The CMS4 subtype, also known as the Mesenchymal subtype, is considered the most difficult to treat and most lethal subtype. This subtype is characterized by stromal infiltration, overexpression of extracellular matrix proteins and high mixture with non-cancer cells. Furthermore, it is associated with the activation of transforming growth factor $\beta$ (TGF $\beta$ ) signaling, angiogenesis, matrix remodeling pathways and complement inflammatory system activation. All of these features correlate with aggressive tumor behavior resulting in poor disease control and prognosis (33). Based on its characteristics, the CMS4 subtype is the most likely subtype to metastasize to distant organs.

Ever since the development of this new classification system, much research on the clinical implications of this CMS classification is performed. Linnekamp et al. investigated in vitro and in vivo drug response to different CMS subtypes and demonstrated that the CMS4 subtype was significantly less sensitive to $5-\mathrm{FU}$ as compared to the other CMS subtypes (34). The same applied to oxaliplatin: CMS4 cell lines were more resistant to oxaliplatin-induced apoptosis compared to other cell lines. This implicates that CMS4 tumors are (partially) chemotherapy-resistant. A clinical study investigating the effects of adjuvant oxaliplatin- 
based chemotherapy demonstrated that patients with CMS4 subtype tumors showed worse prognosis as compared to patients with other subtypes, regardless of clinical tumor stage (35). Hence, both translational and clinical studies have shown that CMS4 tumors are less sensitive to oxaliplatinbased chemotherapy as compared to other subtypes.

\section{CMS4 subtype in the context of peritoneal metastases}

A recent study demonstrated that the CMS4 subtype was highly prevalent in primary tumors of patients presenting with PM (60\%), which was significantly higher than the incidence of CMS4 in all patients with stage I-IV CRC, (23\%, $\mathrm{P}=0.002$ ) (36). More importantly, the majority of PM (75\%) were classified as CMS4. This is significantly higher as compared to the incidence of CMS4 in LM as reported in two other studies ( $47 \%, \mathrm{P}=0.004$ and $46.4 \%, \mathrm{P}=0.007$ ) $(37,38)$.

\section{Consequences of the overrepresentation of CMS4 for the treatment of colorectal PM}

The finding that CMS4 is the predominant subtype in PM plus CMS4 being relavitely resistand to oxaliplatin, as described above, needs to be confirmed in larger future studies. These findings would indeed confirm the longstanding notion amongst experts that PM are resistant to systemic chemotherapy. Interestingly, recent in-vitro studies using patient-derived organoids of colorectal PM also showed oxaliplatin-resistance in doses that are currently used in HIPEC-regimens (39). Besides a very promising tool for individual-patient level testing of drug efficacy prior to HIPEC, this suggests that oxaliplatin might be inefficient during HIPEC.

If overrepresentation of oxaliplatin-resistant CMS4 in colorectal PM will be further confirmed, it may have profound consequences for the treatment of PM. Firstly, the systemic treatment of these patients should be reevaluated as most regimens are currently oxaliplatin-based. Secondly, it would provide—at least in part—an explanation why recent RCTs investigating the efficacy of HIPEC may have failed to show such an effect. In both the French PRODIGE-7 trial and the Dutch COLOPEC-trial, an oxaliplatin-based HIPEC-regimen was used $(30,40)$. This may indeed not be effective in intrinsic oxaliplatin-resistant CMS4-type PM. Thus, not the HIPEC-procedure by itself as tested in these trials but the chemotherapeutic agent used during HIPEC may be ineffective. Future research in the treatment of PM, both systemically and intraperitoneally during HIPEC, should focus on investigating cytotoxic agents specifically towards CMS4-subtype tumors.

\section{The importance of the KRAS/BRAF pathway}

Besides CMS4, mutations occurring in the genome of the PM may be important when considering systemic treatment in these patients. Recently, mutations in the KRAS/ BRAF pathway have been investigated. The BRAF and KRAS proteins act as downstream secondary messengers of the epidermal growth factor receptor (EGFR), which regulates cancer-cell proliferation, apoptosis and tumorinduced neoangiogenesis (41). Anti-EGFR therapy prevents intracellular tyrosine kinase activation and, in that way, it counteracts the activation of KRAS and BRAF proteins. The application of these regimens is proven to be effective in metastatic CRC patients, resulting in improved OS, most effectively in combination with standard cytostatic regimens (42-45). As such, anti-EGFR may also be considered in patients with PM.

However, mutations in these signaling pathways downstream from EGFR may induce pathway activation which is independent of EGFR. As a result, EGFR blockage at the cell surface by EGFR-targeted regimens is ineffective in patients having such mutations (46).

KRAS gene mutations are present in $35-45 \%$ of patients with metastatic CRC $(47,48)$. Furthermore, in patients with the KRAS wildtype, another $40-60 \%$ of patients are non-responders to EGFR-targeted therapy (49). Previous studies suggest this insensitivity could be due to mutations in other genes, like BRAF (46). BRAF mutations are present in $5-10 \%$ of metastatic colorectal tumors $(50,51)$. However, most studies on this subject included mainly patients with colorectal LM (52-58).

A recently published study by Graf et al. (59) demonstrated that KRAS mutations were present in $46 \%$ of patients with colorectal or appendiceal PM. BRAF mutations were present in $11 \%$ of these patients and were associated with worse OS compared to BRAF wildtype tumors. KRAS mutations were not associated with worse OS in this study. Another study presented a higher percentage of BRAF mutations in patients with isolated colorectal PM, compared to patients having systemic metastases of colorectal origin (18\% vs. 9\%) (60). Moreover, in this study, BRAF mutations were not associated with worse OS, as compared to wildtype BRAF patients. A third study also demonstrated impaired OS in KRAS/ 
BRAF mutated tumors as compared to wildtype tumors, and this was regardless the administrated therapy (61). Taken together, mutations in the cancer genome such as in KRAS and BRAF have an important prognostic effect in colorectal PM. Future research should elucidate the mechanisms by which these mutations impair survival, potentially identifying targets for more effective treatment.

\section{Conclusion and future perspective}

There is currently no consensus regarding the value of systemic therapy either in a neoadjuvant setting, adjuvant setting or both in patients with colorectal PM undergoing CRS-HIPEC. Two systematic reviews concluded there is no high-level evidence to support either of the strategies. As such, the decision whether or not to add systemic treatment to CRS-HIPEC remains a matter of expert opinion, resulting in multiple protocols around the world. Ideally, this question should be answered with data from an RCT. Currently, such an RCT is recruiting patients in the Netherlands. The recently published COMBATACtrial illustrated the importance of intention-to-treat analysis to avoid selection bias given the high number of patients that did not proceed to CRS-HIPEC after neoadjuvant treatment. Studies investigating the effect of systemic treatment in combination with CRS-HIPEC that only report on postoperative patients are probably subject to (severe) selection bias, as for instance the recently presented PRODIGE7-trial.

The notion that the majority of PM are probably of the CMS4-subtype and that this subtype is relatively resistant to various cytostatic drugs, including oxaliplatin, sheds new light on currently available systemic and intraperitoneal treatment regimens containing oxaliplatin. New cytotoxic agents specifically targeting CMS4 may further improve treatment of patients with colorectal PM.

Further analysis of specific mutations in the cancer genome of PM may provide important information on the efficacy of modern treatments (e.g., anti-EGFR treatment), may give prognostic information and may identify new targets for treatment.

In the future, systemic treatment of patients with PM will probably become individualized, based on their specific cancer genome and the consensus molecular subtype of their metastases. Advanced in-vitro testing of drug sensitivity prior to start of treatment, such as patient derived organoid technologies, may help to further enhance tailored treatment.

\section{Acknowledgments}

Funding: None.

\section{Footnote}

Provenance and Peer Review: This article was commissioned by the Guest Editors (Paul H. Sugarbaker and Kurt Van der Speeten) for the focused issue "Intraperitoneal Chemotherapy for Peritoneal Metastases: HIPEC, EPIC, NIPEC, PIPAC and More" published in fournal of Gastrointestinal Oncology. This article has undergone external peer review.

Conflicts of Interest: All authors have completed the ICMJE uniform disclosure form (available at http://dx.doi. org/10.21037/jgo-20-133). The focused issue was sponsored by the Peritoneal Surface Oncology Group International (PSOGI). IHJTDH reports a unrestricted grant was paid to the institute by RanD Biotech and QP\&S. The authors have no other conflicts of interest to declare.

Ethical Statement: The authors are accountable for all aspects of the work in ensuring that questions related to the accuracy or integrity of any part of the work are appropriately investigated and resolved.

Open Access Statement: This is an Open Access article distributed in accordance with the Creative Commons Attribution-NonCommercial-NoDerivs 4.0 International License (CC BY-NC-ND 4.0), which permits the noncommercial replication and distribution of the article with the strict proviso that no changes or edits are made and the original work is properly cited (including links to both the formal publication through the relevant DOI and the license). See: https://creativecommons.org/licenses/by-nc-nd/4.0/.

\section{References}

1. Lemmens VE, Klaver YL, Verwaal VJ, et al. Predictors and survival of synchronous peritoneal carcinomatosis of colorectal origin: A population-based study. Int J Cancer 2011;128:2717-25.

2. Klaver YLB, Lemmens VEPP, Nienhuijs SW, et al. Peritoneal carcinomatosis of colorectal origin: Incidence, prognosis and treatment options. World Journal of Gastroenterology 2012;18:5489-94.

3. Segelman J, Granath F, Holm T, et al. Incidence, 
prevalence and risk factors for peritoneal carcinomatosis from colorectal cancer. Br J Surg 2012;99:699-705.

4. Kerscher AG, Chua TC, Gasser M, et al. Impact of peritoneal carcinomatosis in the disease history of colorectal cancer management: A longitudinal experience of 2406 patients over two decades. Br J Cancer 2013;108:1432-9.

5. Segelman J, Akre O, Gustafsson UO, et al. Individualized prediction of risk of metachronous peritoneal carcinomatosis from colorectal cancer. Colorectal Dis 2014;16:359-67.

6. Lahaye M, Lambregts DM, Eden WH Van, et al. Diagnostic value of imaging for the detection of peritoneal metastases: A meta-analysis. United Eur Gastroenterol J 2016;30:3101-12.

7. Verwaal VJ, van Ruth S, de Bree E, et al. Randomized trial of cytoreduction and hyperthermic intraperitoneal chemotherapy versus systemic chemotherapy and palliative surgery in patients with peritoneal carcinomatosis of colorectal cancer. J Clin Oncol 2003;21:3737-43.

8. Verwaal VJ, Bruin S, Boot H, et al. 8-Year follow-up of randomized trial: Cytoreduction and hyperthermic intraperitoneal chemotherapy versus systemic chemotherapy in patients with peritoneal carcinomatosis of colorectal cancer. Ann Surg Oncol 2008;15:2426-32.

9. Simkens GA, van Oudheusden TR, Luyer MD, et al. Serious Postoperative Complications Affect Early Recurrence After Cytoreductive Surgery and HIPEC for Colorectal Peritoneal Carcinomatosis. Ann Surg Oncol 2015;22:2656-62.

10. Feferman Y, Solomon D, Bhagwandin S, et al. Sites of Recurrence After Complete Cytoreduction and Hyperthermic Intraperitoneal Chemotherapy for Patients with Peritoneal Carcinomatosis from Colorectal and Appendiceal Adenocarcinoma: A Tertiary Center Experience. Ann Surg Oncol 2019;26:482-9.

11. van Gestel YR, Thomassen I, Lemmens VE, et al. Metachronous peritoneal carcinomatosis after curative treatment of colorectal cancer. Eur J Surg Oncol 2014;40:963-9.

12. van Gestel YRBM, de Hingh IHJT, van Herk-Sukel MPP, et al. Patterns of metachronous metastases after curative treatment of colorectal cancer. Cancer Epidemiol 2014;38:448-54

13. Passot G, You B, Boschetti G, et al. Pathological response to neoadjuvant chemotherapy: A new prognosis tool for the curative management of peritoneal colorectal carcinomatosis. Ann Surg Oncol 2014;21:2608-14.
14. Passot G, Vaudoyer D, Cotte E, et al. Progression following neoadjuvant systemic chemotherapy may not be a contraindication to a curative approach for colorectal carcinomatosis. Ann Surg 2012;256:125-9.

15. Elias D, Gilly F, Boutitie F, et al. Peritoneal colorectal carcinomatosis treated with surgery and perioperative intraperitoneal chemotherapy: Retrospective analysis of 523 patients from a multicentric French study. J Clin Oncol 2010;28:63-8.

16. Baratti D, Kusamura S, Iusco D, et al. Postoperative complications after cytoreductive surgery and hyperthermic intraperitoneal chemotherapy affect longterm outcome of patients with peritoneal metastases from colorectal cancer: A two-center study of 101 patients. Dis Colon Rectum 2014;57:858-68.

17. Devilee RA, Simkens GA, van Oudheusden TR, et al. Increased Survival of Patients with Synchronous Colorectal Peritoneal Metastases Receiving Preoperative Chemotherapy Before Cytoreductive Surgery and Hyperthermic Intraperitoneal Chemotherapy. Ann Surg Oncol 2016;23:2841-8.

18. Elias D, Lefevre JH, Chevalier J, et al. Complete cytoreductive surgery plus intraperitoneal chemohyperthermia with oxaliplatin for peritoneal carcinomatosis of colorectal origin. J Clin Oncol 2009;27:681-5.

19. Simkens GA, Rovers KP, Nienhuijs SW, et al. Patient selection for cytoreductive surgery and HIPEC for the treatment of peritoneal metastases from colorectal cancer. Cancer Manag Res 2017;9:259.

20. Rovers KP, Simkens GA, Punt CJ, et al. Perioperative systemic therapy for resectable colorectal peritoneal metastases: Sufficient evidence for its widespread use? A critical systematic review. Crit Rev Oncol Hematol 2017;114:53-62.

21. Bushati M, Rovers KP, Sommariva A, et al. The current practice of cytoreductive surgery and HIPEC for colorectal peritoneal metastases: Results of a worldwide webbased survey of the Peritoneal Surface Oncology Group International (PSOGI). Eur J Surg Oncol 2018;44:1942-8.

22. Esquivel J, Sticca R, Sugarbaker P, et al. Cytoreductive surgery and hyperthermic intraperitoneal chemotherapy in the management of peritoneal surface malignancies of colonic origin: A consensus statement. Ann Surg Oncol 2007 14:128-33.

23. Nordlinger B, Sorbye H, Glimelius B, et al. Perioperative chemotherapy with FOLFOX4 and surgery versus surgery alone for resectable liver metastases from colorectal cancer 
(EORTC Intergroup trial 40983): a randomised controlled trial. Lancet 2008;371:1007-16.

24. Nordlinger B, Sorbye H, Glimelius B, et al. Perioperative FOLFOX 4 chemotherapy and surgery versus surgery alone for resectable liver metastases from colorectal cancer (EORTC 40983): Long-term results of a randomised, controlled, phase 3 trial. Lancet Oncol 2013;14:1208-15.

25. Cleeland CS. Symptom burden: multiple symptoms and their impact as patient-reported outcomes. Journal of the National Cancer Institute. Monographs 2007;37:16-21.

26. Glockzin G, Zeman F, Croner RS, et al. Perioperative Systemic Chemotherapy, Cytoreductive Surgery, and Hyperthermic Intraperitoneal Chemotherapy in Patients With Colorectal Peritoneal Metastasis: Results of the Prospective Multicenter Phase 2 COMBATAC Trial. Clin Colorectal Cancer 2018;17:285-96.

27. Eveno C, Passot G, Goéré D, et al. Bevacizumab doubles the early postoperative complication rate after cytoreductive surgery with hyperthermic intraperitoneal chemotherapy (HIPEC) for peritoneal carcinomatosis of colorectal origin. Ann Surg Oncol 2014;21:1792-800.

28. Simkens GA, Rovers KP, Van Oudheusden TR, et al. Major influence of postoperative complications on costs of cytoreductive surgery and HIPEC in patients with colorectal peritoneal metastases. Medicine (Baltimore) 2018;97:e0042.

29. Waite K, Youssef H. The Role of Neoadjuvant and Adjuvant Systemic Chemotherapy with Cytoreductive Surgery and Heated Intraperitoneal Chemotherapy for Colorectal Peritoneal Metastases: A Systematic Review. Ann Surg Oncol 2017;24:705-20.

30. Quenet F, Elias D, Roca L, et al. A UNICANCER phase III trial of hyperthermic intra-peritoneal chemotherapy (HIPEC) for colorectal peritoneal carcinomatosis (PC): PRODIGE 7. J Clin Oncol 2018;36:abstr 3503.

31. Rovers KP, Bakkers C, Simkens GAAM, et al. Perioperative systemic therapy and cytoreductive surgery with HIPEC versus upfront cytoreductive surgery with HIPEC alone for isolated resectable colorectal peritoneal metastases: Protocol of a multicentre, open-label, parralelgroup, phase II-III, random. BMC Cancer 2019;19:390.

32. Guinney J, Dienstmann R, Wang X, et al. The consensus molecular subtypes of colorectal cancer. Nat Med 2015;21:1350-6.

33. Hanahan D, Weinberg RA. Hallmarks of cancer: The next generation. Cell 2011;144:646-74.

34. Linnekamp JF, Van Hooff SR, Prasetyanti PR, et al. Consensus molecular subtypes of colorectal cancer are recapitulated in in vitro and in vivo models. Cell Death Differ 2018;25:616-33.

35. Song N, Pogue-Geile KL, Gavin PG, et al. Clinical outcome from oxaliplatin treatment in stage II/III colon cancer according to intrinsic subtypes: Secondary analysis of NSABP C-07/NRG oncology randomized clinical trial. JAMA Oncol 2016;2:1162-9.

36. Ubink I, van Eden WJ, Snaebjornsson P, et al. Histopathological and molecular classification of colorectal cancer and corresponding peritoneal metastases. Br J Surg 2018;105:e204-11.

37. De Sousa E Melo F, Wang X, Jansen M, et al. Poorprognosis colon cancer is defined by a molecularly distinct subtype and develops from serrated precursor lesions. Nat Med 2013;19:614.

38. Trumpi K, Ubink I, Trinh A, et al. Neoadjuvant chemotherapy affects molecular classification of colorectal tumors. Oncogenesis 2017;6:e357.

39. Ubink I, Bolhaqueiro ACF, Elias SG, et al. Organoids from colorectal peritoneal metastases as a platform for improving hyperthermic intraperitoneal chemotherapy. $\mathrm{Br}$ J Surg 2019;106:1404-14.

40. Klaver CEL, Wisselink DD, Punt CJA, et al. Adjuvant hyperthermic intraperitoneal chemotherapy in patients with locally advanced colon cancer (COLOPEC): a multicentre, open-label, randomised trial. Lancet Gastroenterol Hepatol 2019;4:761-70.

41. Normanno N, Tejpar S, Morgillo F, et al. Implications for KRAS status and EGFR-targeted therapies in metastatic CRC. Nat Rev Clin Oncol 2009;6:519.

42. Ciardiello F, Tortora G. Drug therapy: EGFR antagonists in cancer treatment. N Engl J Med 2008;358:1160-74.

43. Folprecht G, Lutz MP, Schöffski P, et al. Cetuximab and irinotecan/5-fluorouracil/folinic acid is a safe combination for the first-line treatment of patients with epidermal growth factor receptor expressing metastatic colorectal carcinoma. Ann Oncol 2006;17:450-6.

44. Tabernero J, Van Cutsem E, Díaz-Rubio E, et al. Phase II trial of cetuximab in combination with fluorouracil, leucovorin, and oxaliplatin in the first-line treatment of metastatic colorectal cancer. J Clin Oncol 2007;25:5225-32.

45. Van Cutsem E, Nowacki M, Lang I, et al. Randomized phase III study of irinotecan and 5-FU/FA with or without cetuximab in the first-line treatment of patients with metastatic colorectal cancer (mCRC): The CRYSTAL trial. J Clin Oncol 2007;25:abstr 4000.

46. Therkildsen C, Bergmann TK, Henrichsen-Schnack T, et al. The predictive value of KRAS, NRAS, BRAF, PIK3CA 
and PTEN for anti-EGFR treatment in metastatic colorectal cancer: A systematic review and meta-analysis. Acta Oncologica 2014;53:852-64.

47. Allegra CJ, Jessup JM, Somerfield MR, et al. American society of clinical oncology provisional clinical opinion: Testing for KRAS gene mutations in patients with metastatic colorectal carcinoma to predict response to antiepidermal growth factor receptor monoclonal antibody therapy. J Clin Oncol 2009;27:2091-6.

48. Van Cutsem E, Köhne CH, Láng I, et al. Cetuximab plus irinotecan, fluorouracil, and leucovorin as firstline treatment for metastatic colorectal cancer: Updated analysis of overall survival according to tumor KRAS and BRAF mutation status. J Clin Oncol 2011;29:2011-9.

49. Linardou H, Dahabreh IJ, Kanaloupiti D, et al. Assessment of somatic k-RAS mutations as a mechanism associated with resistance to EGFR-targeted agents: a systematic review and meta-analysis of studies in advanced non-smallcell lung cancer and metastatic colorectal cancer. Lancet Oncol 2008;9:962-72.

50. Douillard JY, Oliner KS, Siena S, et al. PanitumumabFOLFOX4 treatment and RAS mutations in colorectal cancer. N Engl J Med 2013;369:1023-34.

51. Berlin J. Beyond exon 2 - The developing story of RAS mutations in colorectal cancer. N Engl J Med 2013;369:1059-60.

52. Umeda Y, Nagasaka T, Mori Y, et al. Poor prognosis of KRAS or BRAF mutant colorectal liver metastasis without microsatellite instability. J Hepatobiliary Pancreat Sci 2013;20:223-33.

53. Tian S, Simon I, Moreno V, et al. A combined oncogenic pathway signature of braf, KRAS and PI3KCA mutation improves colorectal cancer classification and cetuximab treatment prediction. Gut 2013;62:540-9.

54. Zhang J, Zheng J, Yang Y, et al. Molecular spectrum of

Cite this article as: Bakkers C, Simkens GAAM, De Hingh IHJT. Systemic therapy in addition to cytoreduction and hyperthermic intraperitoneal chemotherapy for colorectal peritoneal metastases: recent insights from clinical studies and translational research. J Gastrointest Oncol 2021;12 (Suppl 1):S206-S213. doi: 10.21037/jgo-20-133
KRAS, NRAS, BRAF and PIK3CA mutations in Chinese colorectal cancer patients: Analysis of 1,110 cases. Sci Rep 2015;5:18678.

55. Fariña-Sarasqueta A, van Lijnschoten G, Moerland E, et al. The BRAF V600E mutation is an independent prognostic factor for survival in stage II and stage III colon cancer patients. Ann Oncol 2010;21:2396-402.

56. Løes IM, Immervoll H, Sorbye H, et al. Impact of KRAS, BRAF, PIK3CA, TP53 status and intraindividual mutation heterogeneity on outcome after liver resection for colorectal cancer metastases. Int J Cancer 2016;139:647-56.

57. Roth AD, Tejpar S, Delorenzi M, et al. Prognostic role of KRAS and BRAF in stage II and III resected colon cancer: Results of the translational study on the PETACC-3, EORTC 40993, SAKK 60-00 trial. J Clin Oncol 2010;28:466-74.

58. Tol J, Nagtegaal ID, Punt CJA. BRAF mutation in metastatic colorectal cancer. N Engl J Med 2009;361:98-9.

59. Graf W, Cashin PH, Ghanipour L, et al. Prognostic Impact of BRAF and KRAS Mutation in Patients with Colorectal and Appendiceal Peritoneal Metastases Scheduled for CRS and HIPEC. Ann Surg Oncol 2020;27:293-300.

60. Franko J, Shi Q, Meyers JP, et al. Prognosis of patients with peritoneal metastatic colorectal cancer given systemic therapy: an analysis of individual patient data from prospective randomised trials from the Analysis and Research in Cancers of the Digestive System (ARCAD) database. Lancet Oncol 2016;17:1709-19.

61. Schneider MA, Eden J, Pache B, et al. Mutations of RAS/ $\mathrm{RAF}$ proto-oncogenes impair survival after cytoreductive surgery and HIPEC for peritoneal metastasis of colorectal origin. Ann Surg 2018;268:845-53. 